\title{
Unusual milia-type intradermal tophi in a patient with gout
}

\author{
Mingyo Kim, Sang-Il Lee, and Yun-Hong Cheon
}

Division of Rheumatology, Department of Internal Medicine, Gyeongsang National University Hospital, Jinju, Korea
Received: September 28, 2018

Revised : October 3, 2018

Accepted: October 4, 2018

\section{Correspondence to}

Yun-Hong Cheon, M.D.

Tel: +82-55-750-9585

Fax: +82-55-758-9122

E-mail: hong369c@naver.com
A 34-year-old man presented with severe pain in multiple joints. He was diagnosed with gout 4 years ago; however, he had not been administered any medication for this condition. He was obese (body mass index, $36.3 \mathrm{~m}^{2} / \mathrm{kg}$ ), and laboratory tests showed increased levels of uric acid (11.4 mg/dL). Physical examination revealed severe edema and pain in the joints with tophi in the following sites: the right first metatarsophalangeal, both lateral malleoli, distal interphalangeal, proximal interphalangeal, and metacarpophalangeal joints and ears (Fig. 1). Following administration of prednisolone and colchicine, the pain improved. Despite the need for continuous treatment, he did not return to our clinic until a year later. He revisited our clinic with multiple painful and itchy yellowish papules on the anterior and posterior pretibial areas (Fig. 2A). Before visiting our clinic, he had been diagnosed with acne or folliculitis and had been prescribed a topical steroid ointment at a local clinic; however, the pain had not alleviated. We performed a dual-energy computed tomography (DECT) and skin biopsy. The DECT showed increasing signals in the left anterior and right posterior pretibial areas, suggestive of tophi (Fig. 2B). The skin-biopsy examination showed dispersed birefringence with fine needle shaped crystals and negative birefringence (Fig. $2 \mathrm{C}$ ). Based on these findings, the patient was diagnosed with miliarial gout with classical tophi.

Classical tophi tend to develop in the joints and surrounding tissues during the most advanced clinical stage of gout. Miliarial gout is an uncommon subcutaneous or interdermal presen- 

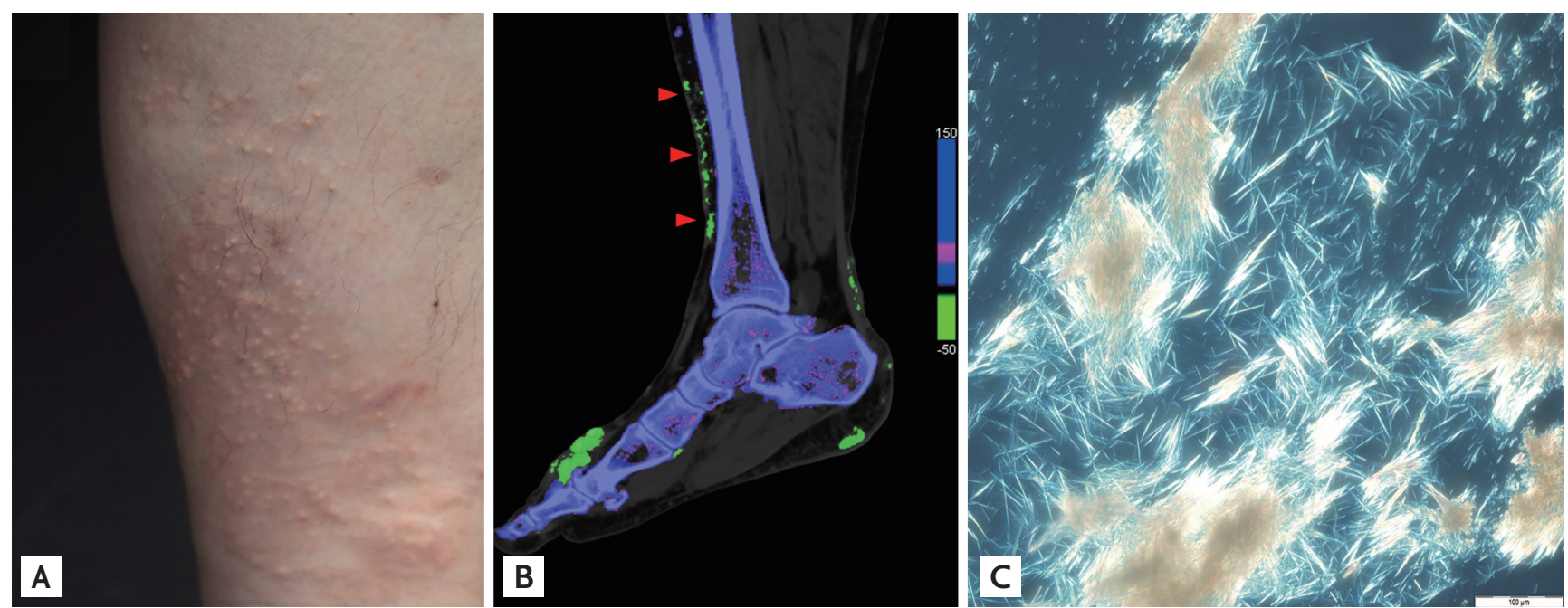

Figure 2. Unusual milia-type intradermal tophi. (A) Milia-type tophi on the anterior pretibial area. The skin-biopsy performed in the anterior pretibial area. (B) Dual-energy computed tomography demonstrating monosodium crystal deposits on the first metatarsophalangeal joint, Achilles tendon, and dermis of the pretibial area (arrowheads). (C) Polarization microscopy of the skin-biopsy sample showed dispersed birefringence fine needle shape crystals and negative birefringence.

tation of gout, developing predominantly in the lower extremities, before or after the development of classic tophaceous gout. The possible pathogenesis of miliarial gout is unclear; however, obesity, chronic venous insufficiency, and long-term use of glucocorticoid are considered risk factors. As in our case, DECT might be a useful tool for diagnosis.

Informed consent was obtained from a patient.

\section{Conflict of interest}

No potential conflict of interest relevant to this article was reported. 